\title{
COMPARING TIME-OF-FLIGHT AND PHASE-SHIFT. THE SURVEY OF THE ROYAL PANTHEON IN THE BASILICA OF SAN ISIDORO (LEÓN)
}

\author{
San José Alonso, J.I., Martínez Rubio, J., Fernández Martín, J.J., García Fernández, J. \\ Laboratory of Architectural Photogrammetry (http://www.uva.es/davap) \\ ETS Arquitectura, Av. De Salamanca s/n 47014 Valladolid. Spain \\ jesussanjose@telefonica.net; juanjo@ega.uva.es,jmrubio@ega.uva.es
}

KEY WORDS: Scanner comparison, accuracy, inspection, polychromies, translucency, apparent micro-relief

\begin{abstract}
:
The appearance of the Terrestrial Laser Scanners or 3D Scanners in Heritage recording has been relatively recent and it is submitted to a constant evolution determined mainly by the big technological advance in fields like Optics, Signal Processing, Electronics and Computer Science. As they have become popular so suddenly, it is essential to study the behavior of these evolving devices in a variety of scenarios to support an accurate assessment of their capabilities.

Until two years ago, TOF (time-of-flight) and PS (phase-shift) technologies could hardly be considered side by side comparable, at least under equal terms and requirements. The first enables much longer ranges, while the latter dominated the short distances producing more accurate data with very high acquisition rates. Today, in a sort of convergent career, the scope of phase-shift technology has grown to near 200 meters and the time-of-flight team have been increasing their speed to figures as 100,000 points per second. In this article we expose the results of the comparison between the data delivered by two scanners based on the two related technologies that categorize today's both long and medium-range scanners. The two have been opposed face to face in the survey of the so called "the Sistine Chapel of the Spanish Romanesque" during the same day, and under the same environmental conditions, using equivalent capture settings.

But now that as we noted these technologies can fight in the same arena, can we claim to be able to produce similar results whatever which one we choose? The answer is "no" or a "conditioned yes" at least. Let's leave numbers and nominal specifications behind and see what else makes them behave so differently.
\end{abstract}

\section{PRESENTATION: THE SCANNER LASER FOR THE TANGIBLE CULTURAL HERITAGE RECORDING.}

The possibility of obtaining a cloud of points with millimeter precision for either large or small surfaces, and the ease of doing surveys aimed not only to planimetric recording but also to $3 \mathrm{D}$ rendering have turn the Terrestrial Laser Scanner into a tool of great potential in Tangible Cultural Heritage documentation, specifically in architectural and urban contexts. The raw product of all laser scanners are the point clouds, they represent the object's surface by a discrete sampling of 3D pixels captured by different principles of measurement. The accuracy of this technology will rely not only on the resolution of discrete points that are generated, but also the ability to interpret correctly the objective surface topography. This study documents and analyses the geometric differences of two scanners (fig.1) with different principles of operation under the same shooting conditions.

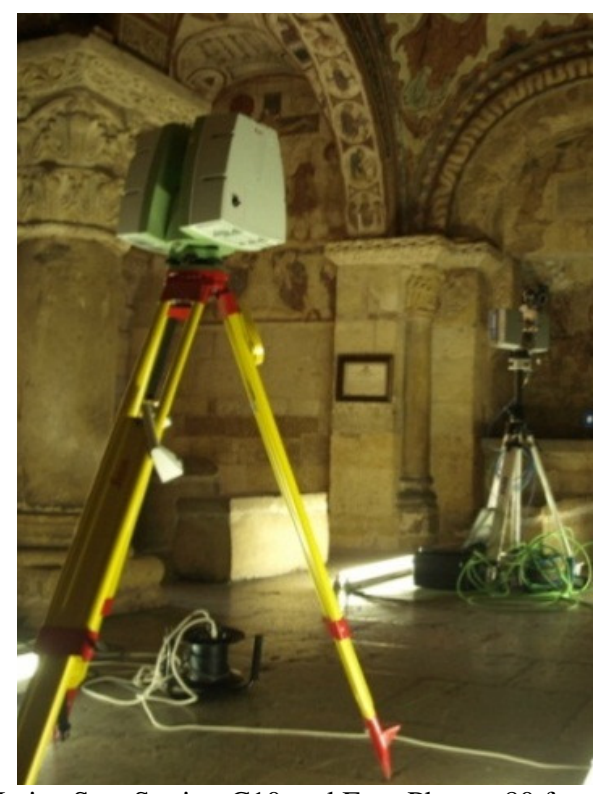

Fig 1. Leica ScanStation C10 and Faro Photon 80 face to face. 


\section{CASE OF STUDY: PANTHEON OF KINGS IN SAN ISIDORO, DOCUMENTATION WITH FARO PHOTON Y LEICA SCANSTATION C10.}

The site for this study is the Pantheon of Kings in San Isidoro (León, Spain). This unique architectural space of the Romanesque style, was designed as the resting place of the remains of the Kings of Leon. Located inside the Basilica of San Isidoro, presented as characteristic features capitals with iconographic motifs (the first in the Spanish Romanesque reproducing scenes from the Gospels), and the paintings in fresco, done in the twelfth century on the vaults, arches and walls, which have earned for the Pantheon the sobriquet "The Sistine Chapel of the Romanesque". The building materials are mainly natural stone and marble quarrying, while the pictorial decorations are preserved almost in perfect condition without any external intervention on the stone composed of colored pigments diluted in water on a layer of lime plaster.

For data collection two laser scanners were used, Faro Photon 80 and Leica ScanStation C10, that were operated simultaneously to prevent differences in the atmospheric parameters of temperature, humidity and pressure for both data capture.

\section{FARO PHOTON 80 LASER SCANNER: WORKING PRINCIPLE AND SCANNING SETTINGS FOR THE CASE OF STUDIO.}

The tls (Terrestrial Laser Scanner) Photon 80 made by Faro, uses the phase-shift principle comparing the phase of the laser source with the same when the radiation comes back again to the scanner after its reflection on object's surface. This type of tls emit continuously a periodical signal of moderate intensity.

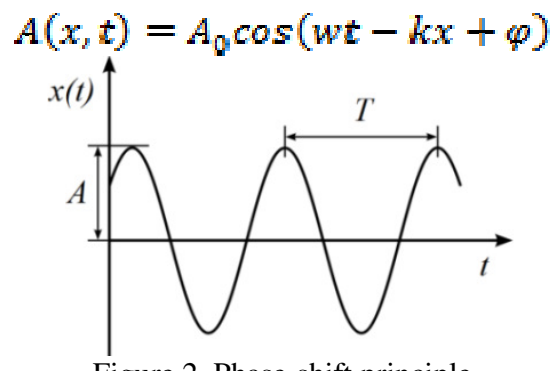

Figure 2. Phase-shift principle

The accuracy of the measurement will be influenced by the length of the cycle of periodicity and also by the wave-length itself that introduce ambiguity in the range estimation. That's why several frequencies are used (multi-frequency-ranging or MF), being the higher to calculate range and the lower to eliminate ambiguity. The pointcloud that arises from the ranging scanner can be colorized by means of a color sensor (typically a digital camera) by fusing both the image and the range data. The technical specifications for the device that we have tested are the following.

Accuracy (referred to one sigma): Uncertainity $\pm 2 \mathrm{~mm}$ at $25 \mathrm{~m}$ distance.

Resolution: Up to 700 millions of points per scan. Effective range: 0.6 to $76 \mathrm{~m}$

Beam type: Near Infrared (wavelength $=785 \mathrm{~nm}$ )

Data capture speed: up to $120.000 \mathrm{pps}$

Field of view: $360^{\circ}$ horizontal by $320^{\circ}$ vertical.

Beam diameter: $3.3 \mathrm{~mm}$ at output.
The scan settings for the case of studio were established to $1 / 5$ of the full resolution (this scanner does not allow to set resolution in terms of point spacing at a particular distance, but just to choose among a few fixed fractions of the full resolution), this is calculated to mean a step of $7 \mathrm{~mm}$ at $10 \mathrm{~m}$. Considering that the ranges we have recorded were shorter, we have got a spot spacing of about $4 \mathrm{~mm}$. The camera was mounted on top of the scanner in "high" mode, which means that the camera fov included the zenith leaving a $50^{\circ}$ cone under the scanner uncovered by photographs.

\section{LEICA SCAN STATION C10: WORKING PRINCIPLE AND SCAN SETTINGS.}

The ScanStation C-10 by Leica-Geosystems uses the LIDAR "time of flight" principle for ranging. A short laser pulse is emitted towards the object and is reflected on its surface; a part of the reflected radiation comes back to the scanner where it is detected by a sensor. As the light-speed is well known and time elapsed between emission and reception of the pulse can be measured (or inferred from a different magnitude measurement), the range to de object can be determined by half of the round-trip distance.
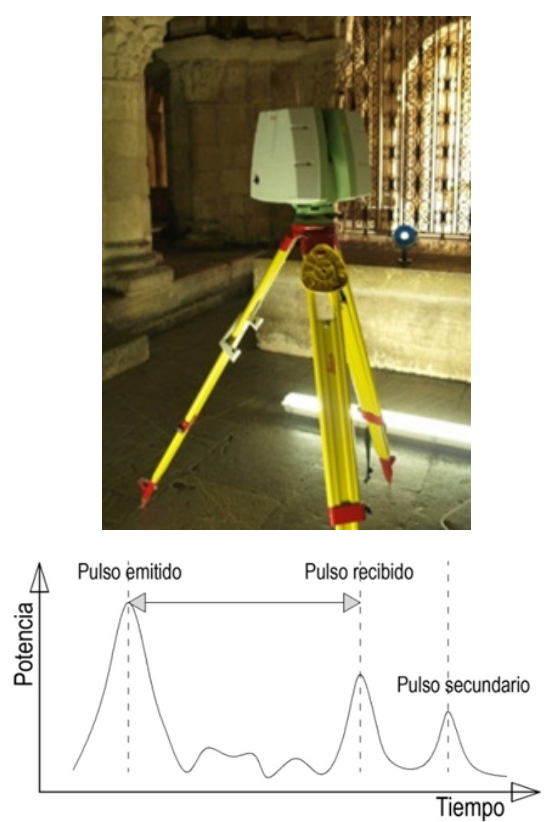

Figure 3. Pulse round-trip timing as ranging principle in TOF

The technical specifications for the $\mathrm{C}-10$ are:

Accuracy referred to one sigma: uncertainty $\pm 4 \mathrm{~mm}$ within 1 50m range.

Resolution: user selectable from less than $1 \mathrm{~mm}$ within the whole range.

Effective range: 0.1 to $300 \mathrm{~m}$

Beam type: Visible Green laser of 532nm wavelength.

Measurement rate: up to 50.000 pps.

Amplitud de giro: $360^{\circ}$ en horizontal y $270^{\circ}$ en vertical

Field of view: $360^{\circ}$ horizontal by $270^{\circ}$ vertical.

Diámetro del haz láser: 4,5mm (basado en $\mathrm{FWHH}$ ); $7 \mathrm{~mm}$ (basado en Gaussian) hasta $50 \mathrm{~m}$ de distancia.

Beam diameter: $4.5 \mathrm{~mm}$ at output and $7 \mathrm{~mm}$ at $50 \mathrm{~m}$ 


\section{COMPARING DATASETS: FARO VS LEICA}

For a more effective comparison and better understanding of the differences between the two records, we have faced the data collected by both scanners in the following subprojects:

1. Head

2. Column

3. Shaft

4. A column sequence

5. Engraved Tablet

6. Wall face

7. Aisle

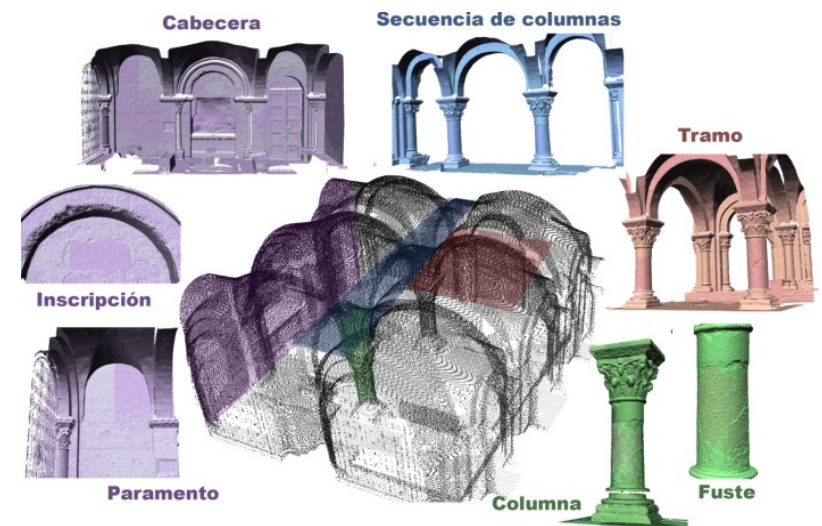

Figure 4. Subprojects.

For the data inspection the software Polyworks has been used. Firstly the scans were parsed from their proprietary native formats to ptx, which is a text format for exchanging scan data that is supported by most vendors. The two datasets each of which was composed of 6 scans had to be aligned and merged and meshed independently using the very same settings. The procedure starts with importing scans into the alignment module, and then processing the result with the meshing and merging one.

\begin{tabular}{|l|l|}
\hline Malign & IMMerge \\
\hline Interpolation Step: 0.01 & Smoothing level: none \\
\hline Max angle: 85 & Meshing/ Max distance: 0.02 \\
\hline Interpolation Step: 0.01 & Meshing/ Surface sampling step: \\
& 0.01 \\
\hline Max angle: 85 & Meshing/ Standard desviation: \\
& 0.0015 \\
\hline Max edge lenght: 0.04 & Reduction tolerance \& \\
& Smoothing: none \\
\hline Subdivision angle: 20 & 3D imagen transitions: Blend \\
\hline
\end{tabular}

Table1. Data processing parameters (for Polyworks)

The two resulting polygonal mesh models were aligned by the IMInspect module, fixing as anchor the one that came out from the $\mathrm{C}-10$ that has served as reference in the further comparison process.

\subsection{Column sequence}

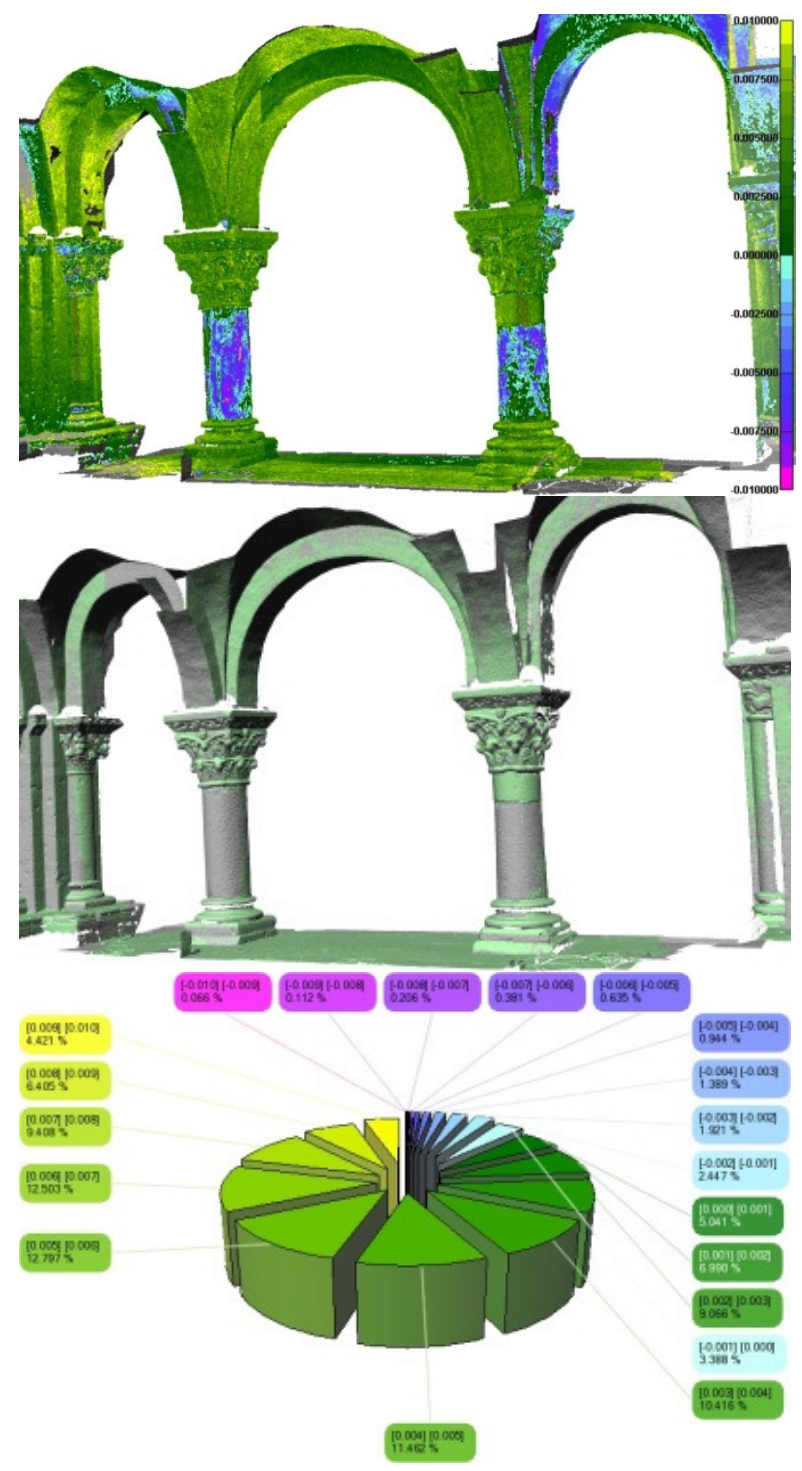

Figure 5. Error-map color mode, per scanner color mode and pie-type error distribution chart for subproject 1.

- $\quad$ ICP Alignment (with a max search-distance of $0.01 \mathrm{~m}$ ) stopped after 5 iterations. The convergence reported was below $7.85 \mathrm{~nm}$

- Colours represent distance between the two meshes measured along normals to reference. The colour scale is set to show distances from -0.01 to +0.01

- Number of points compared 2.320.503

- Average distance. $0.004454 \mathrm{~m}$

- Standard deviation: $0.003771 \mathrm{~m}$

- RMS $0.005836 \mathrm{~m}$

- $99.85 \%$ points within 4 times StdDev. 


\subsection{Aisle}

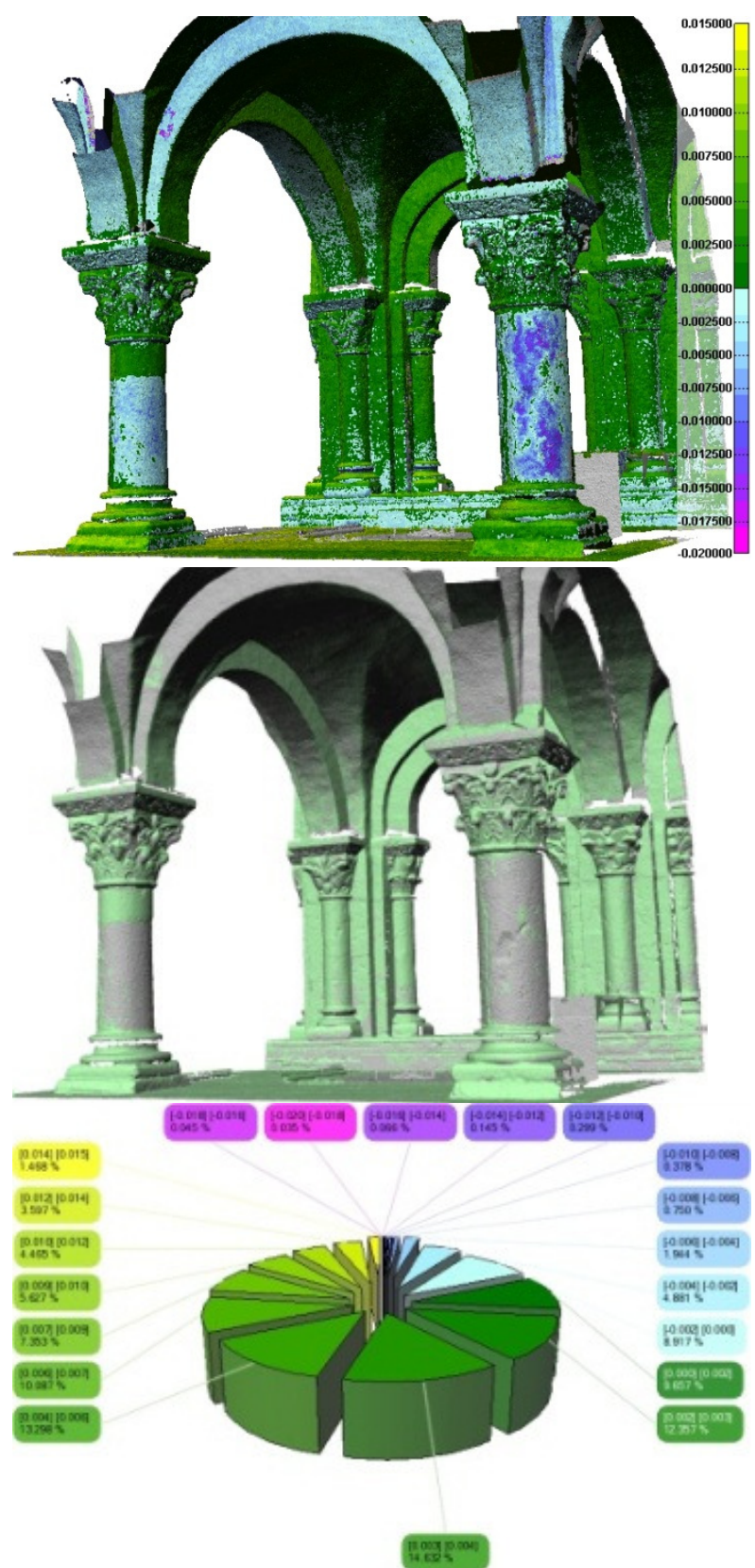

Figure 6. Error-map color mode, per scanner color mode and pie-type error distribution chart for subproject 2.

- ICP Alignment (with a max search-distance of $0.01 \mathrm{~m}$ ) stopped after 4 iterations. The convergence reported was below $1.14 \mathrm{~nm}$

- Colors represent distance between the two meshes measured along normals to reference. The color scale is set to show distances from -0.02 to +0.015

- Number of points compared 1.408.250

- Average distance. $0.004656 \mathrm{~m}$

- Standard deviation: $0.015374 \mathrm{~m}$

- RMS $0.016064 \mathrm{~m}$

- $99.18 \%$ points within 5 times StdDev.

\subsection{Head}
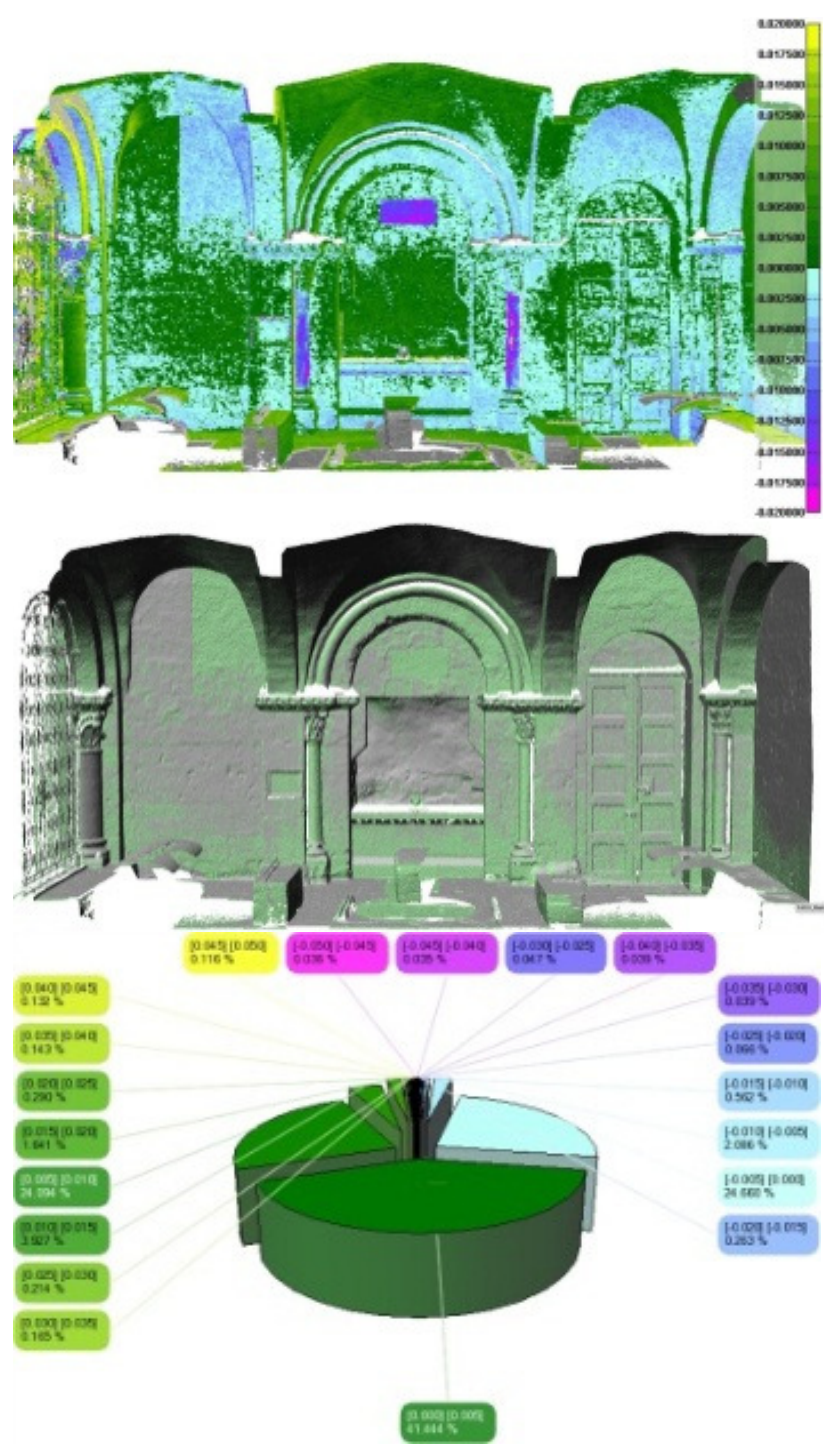

Figure 7. Error-map color mode, per scanner color mode and pie-type error distribution chart for subproject 3.

- $\quad$ ICP Alignment (with a max search-distance of $0.01 \mathrm{~m}$ ) stopped after 4 iterations. The convergence reported was below $1.19 \mathrm{~nm}$

- Colors represent distance between the two meshes measured along normals to reference. The color scale is set to show distances from -0.02 to +0.02

- Number of points compared 1.740 .776

- Average distance. $0.0029 \mathrm{~m}$

- Standard deviation: $0.004974 \mathrm{~m}$

- RMS $0.006668 \mathrm{~m}$

- $99.06 \%$ points within 4 times StdDev. 


\subsection{Single column}

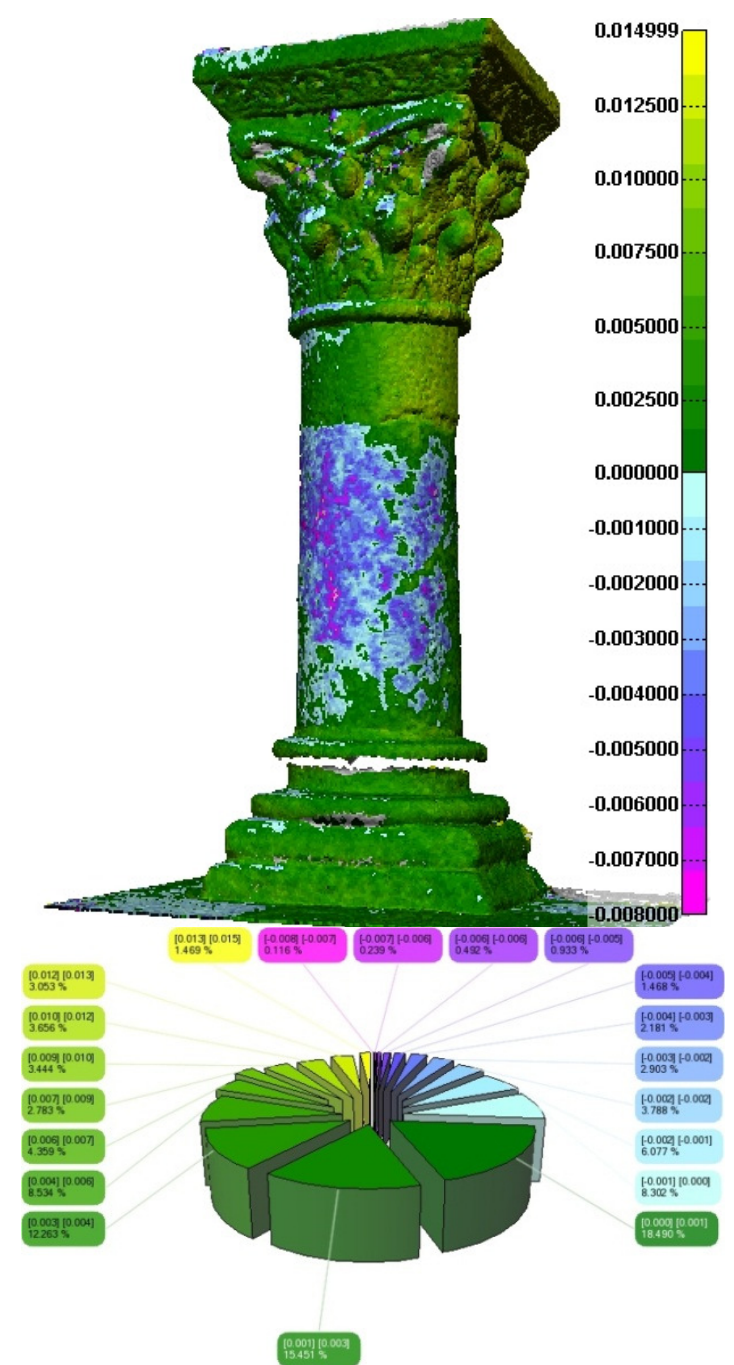

Figure 8. Error-map color mode and pie-type error distribution chart for subproject 4.

- ICP Alignment (with a max search-distance of $0.01 \mathrm{~m}$ ) stopped after 6 iterations. The convergence reported was below $0.216 \mathrm{~nm}$

- Colors represent distance between the two meshes measured along normals to reference. The color scale is set to show distances from -0.008 to +0.015

- Number of points compared 261.864

- Average distance. $0.002708 \mathrm{~m}$

- Standard deviation: $0.004369 \mathrm{~m}$

- $\quad$ RMS $0.005140 \mathrm{~m}$

- $99.89 \%$ points within 3 times StdDev.

\subsection{Column shaft}

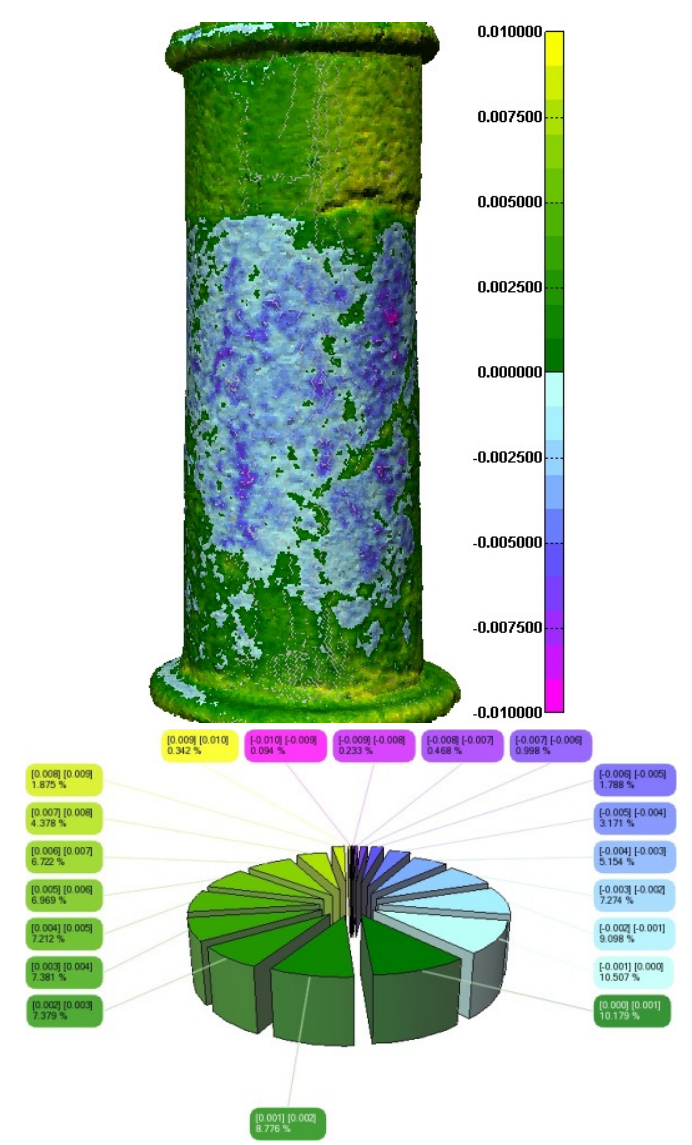

Figure 9. Error-map color mode and pie-type error distribution chart for subproject 5 .

- ICP Alignment (with a max search-distance of $0.01 \mathrm{~m}$ ) stopped after 6 iterations. The convergence reported was below $0.078 \mathrm{~nm}$

- Colors represent distance between the two meshes measured along normals to reference. The color scale is set to show distances from -0.010 to +0.010

- $\quad$ Number of points compared 88.358

- Average distance. $0.003703 \mathrm{~m}$

- Standard deviation: $0.003948 \mathrm{~m}$

- $\quad$ RMS $0.003948 \mathrm{~m}$

- $\quad 99.91 \%$ points within 3 times StdDev.

\subsection{Engraved Tablet}

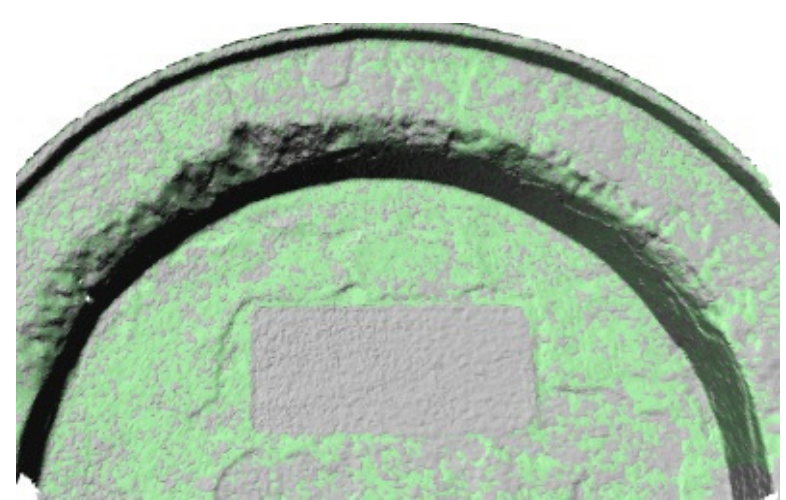

Figure 10. Per Scanner color mode rendering. Subproject 6. 


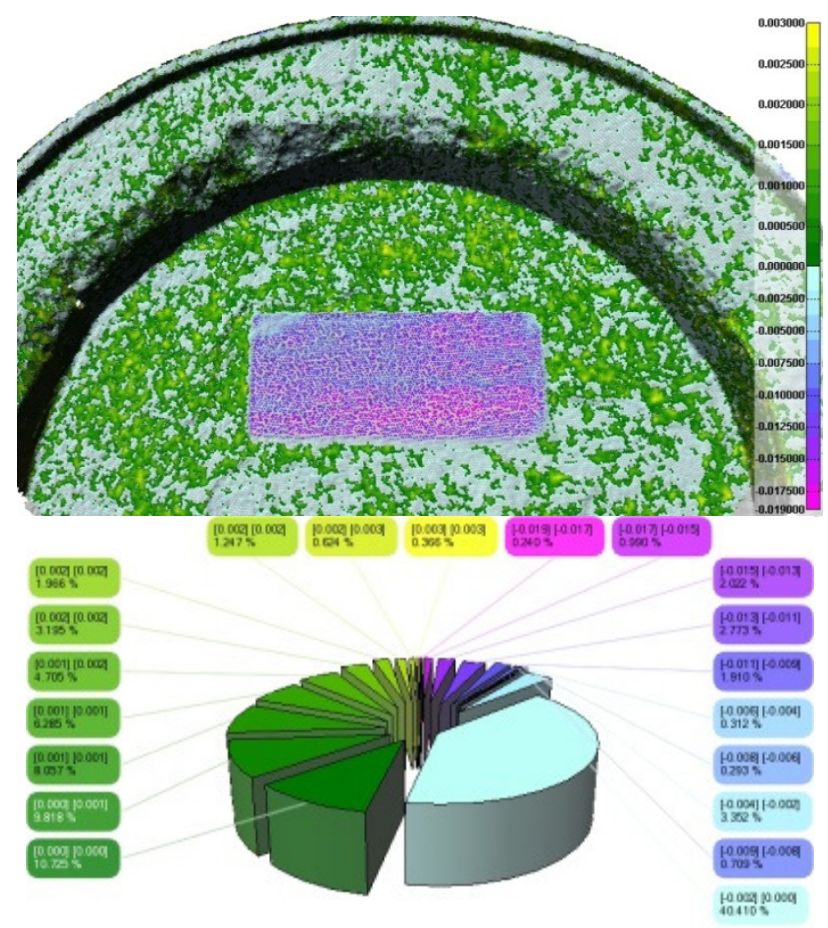

Figure 11. Error map and pie chart for subproject 6

- $\quad$ ICP Alignment (with a max search-distance of $0.01 \mathrm{~m}$ ) stopped after 9 iterations. The convergence reported was below $0.210 \mathrm{~nm}$

- Colors represent distance between the two meshes measured along normals to reference. The color scale is set to show distances from -0.02 to +0.003

- Number of points compared 61.515

- Average distance. $0.001068 \mathrm{~m}$

- Standard deviation: $0.003807 \mathrm{~m}$

- $\quad$ RMS $0.003954 \mathrm{~m}$

- $99.47 \%$ points within 4 times StdDev.

\subsection{Wall face}

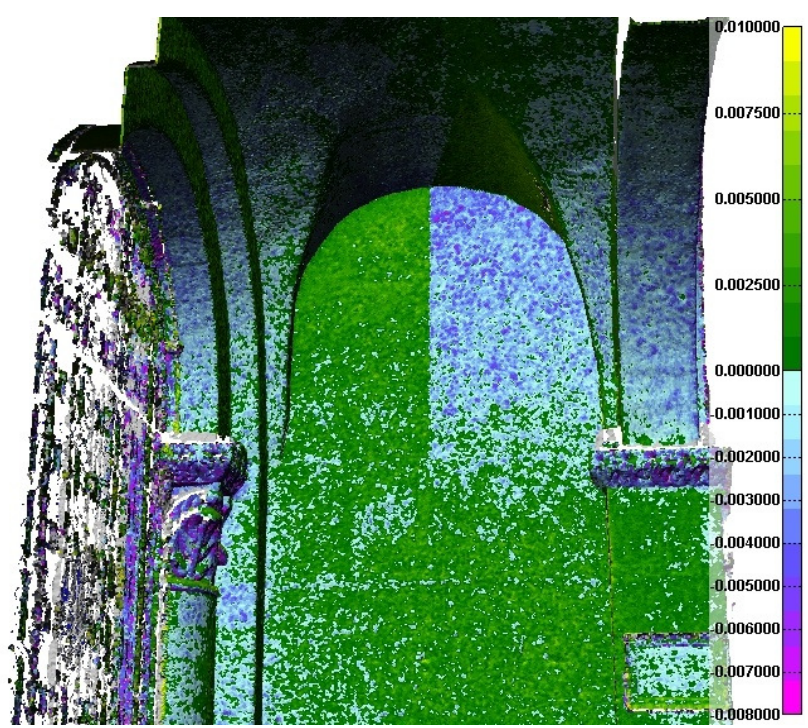

Figure 12. Error map for subproject 7 showing a turning-break

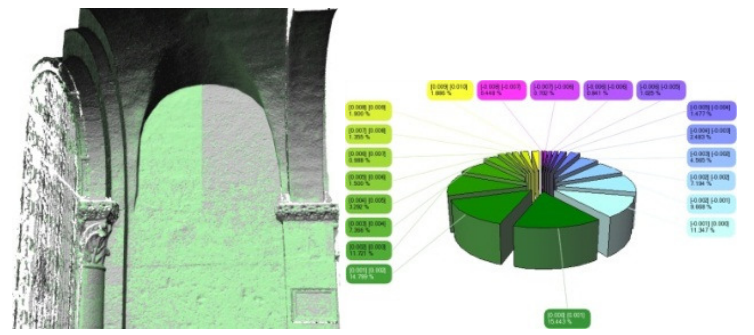

Figure 13. Per mesh color and error pie-chart for sp. 7.

- ICP Alignment (with a max search-distance of $0.01 \mathrm{~m}$ ) stopped after 4 iterations. The convergence reported was below $0.33 \mathrm{~nm}$

- Colors represent distance between the two meshes measured along normals to reference. The color scale is set to show distances from -0.008 to +0.010

- Number of points compared 534.398

- Average distance. $0.002784 \mathrm{~m}$

- Standard deviation: $0.002212 \mathrm{~m}$

- RMS $0.002230 \mathrm{~m}$

- $99.06 \%$ points within 4 times StdDev.

\section{DIFFERENCES FOUND AND THEIR POSSIBLE CAUSE.}

The comparison of data obtained from both scanners show areas with high geometric differences, which implies poor accuracy in one or both instruments under rather common shooting conditions; problems that we consider associated with the physical properties of scanned materials. The main differences and their possible causes are as follows:

\subsection{Differences in topography and noise in the data on marble elements:}

Marble is an inhomogeneous material whose main component of calcium carbonate $(\mathrm{CaCO} 3)$ is present in the form of crystallized calcarenites (approximately $90 \%$ of the total material), and features in different concentrations impurities or inclusions of other minerals which cause differences in color and other properties for the diverse and spread variety.

Due to its composition, the marble exhibits optical peculiarities affecting data capture with laser scanners of objects that are made of such material, these are:

- Translucency. Very important in samples with a high prevalence of calcarenites whose refractive index between 1.49-1.66 is small enough to let in light.

- Anisotropy. The inhomogeneous aggregation of crystals can affect the reflections and transmission of the beam inside the material.

- Chromatism: different colours and its distribution in streaks or patches, can influence the geometry gathered by the scanner.

Most materials, especially the ones involved in Architectural and Urban heritage, are opaque enough to avoid the light to penetrate too much when obtaining a digital 3D representation. In the case of marble, given the refraction index of its main component, the light wave emitted by the laser scanner Faro Photon does penetrate into the material. The heterogeneous composition eliminates the possibility of a constant or typical penetration of the light beam, so the mistake depends not only on the translucency of the marble but also on the direction of the 
pulse, the shooting distance, the beam print size, etc. The reflected wave train received by the sensor and containing enough information to compare it with the emitted pulse, will be the one which will establish the coordinates of the point.

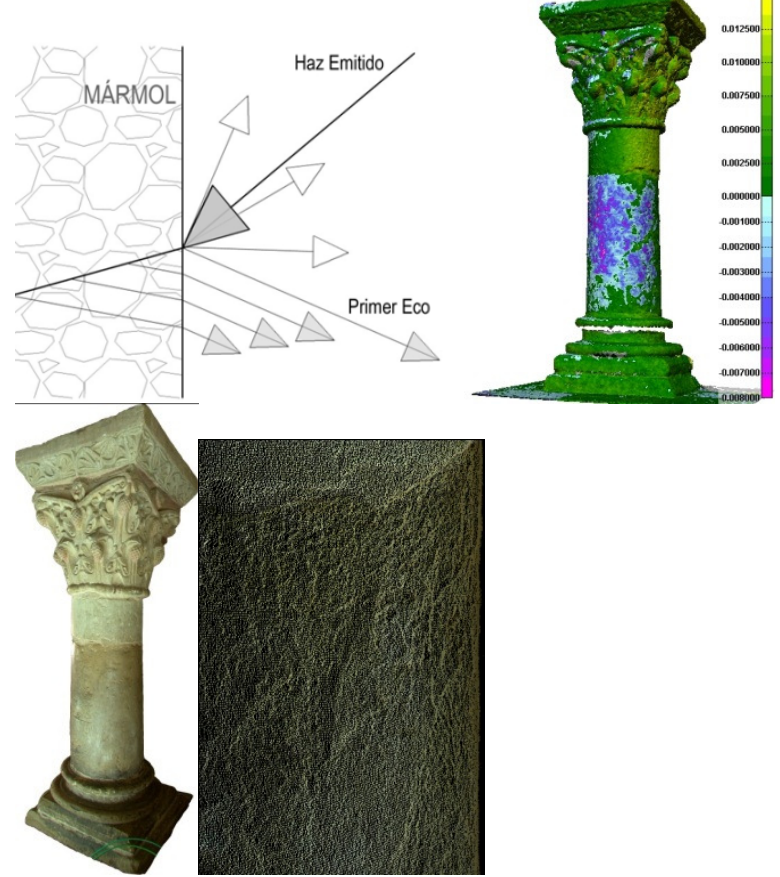

Figure 14. The light permeability issue.

Nowadays there are multi-.pulse laser scanning systems that allows receiving more than one echo; they can distinguish among 6 different ones. Leica C10 cannot do that, it computes just the first return of the emitted pulse. This makes that once the beam has interacted with the surface, it returns with enough energy for the scanner sensor to evaluate it and calculate the coordinates of the $3 \mathrm{D}$ point. This way there is no mistake due to the translucency of the marble. For other scanners, where the evaluated pulse is not the first, an error will be introduced because of the change of velocity when changing from one material to the other (Snell's law).

In the studied case, the surface surveyed with Faro Photon laser scanner presents deviations up to $1 \mathrm{~cm}$ in relation with the data provided by Leica $\mathrm{C} 10$, transforming the smooth and polished marble into a surface with marked roughness.

Related to the surface previously mentioned, the marble presents an increase of the noise in its data. For a general surface documentation, a 3D coordinate summarizes the measurement of the area touched by the laser beam. The heterogeneous composition of materials with different optical properties implies that each point will be calculated as an average material, introducing therefore some noise on each evaluation. This noise will depend both on the scale of the heterogeneous micro-regions and on the size of the laser beam print, defined by the pulse diameter, the shooting distance, the obliquity... etc. The image shows the noise provided by the Faro scanner between the natural stone and the marble.

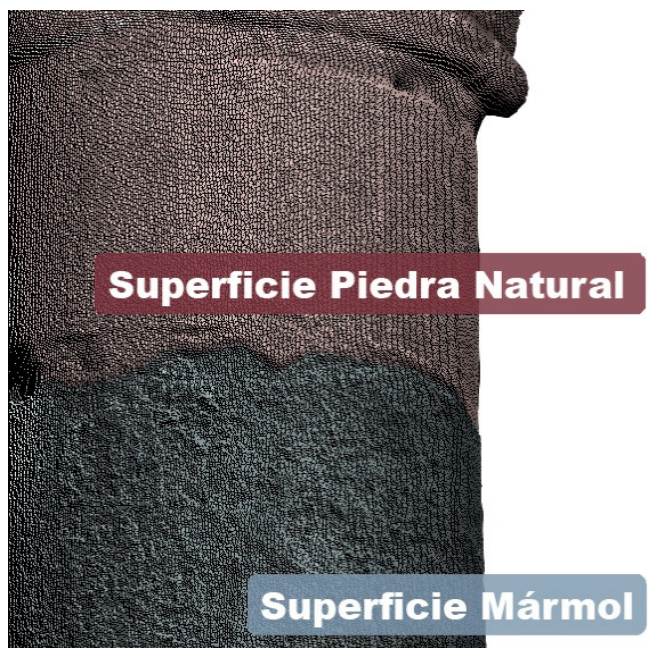

Figure 15. Despite of the model appearance, the finish of the entire shaft is the same for both stones.

\subsection{Scale differences}

The comparative research between data obtained with Faro Photon scanner and Leica C10 shows the existence of scale differences. Interpreting each pair of captures individually, we can observe that data provided by Faro Photon present a larger scale, so the model obtained is smaller than the similar one obtained with the Leica scanner.

The comparison of both data was performed with the IMInspect module of Polyworks software. The alignment was conducted in two parts: firstly a manual approximation, by introducing several pairs of tie points, and then an automatic one, using the "Best Fit" tool, with maximum distances of comparison between $0.1-0.01 \mathrm{~m}$ and maximum angle of $45^{\circ}$. The adjustment was repeated until obtaining convergences ranging from $7.85 \mathrm{~nm}-78.4 \mu \mathrm{m}$.

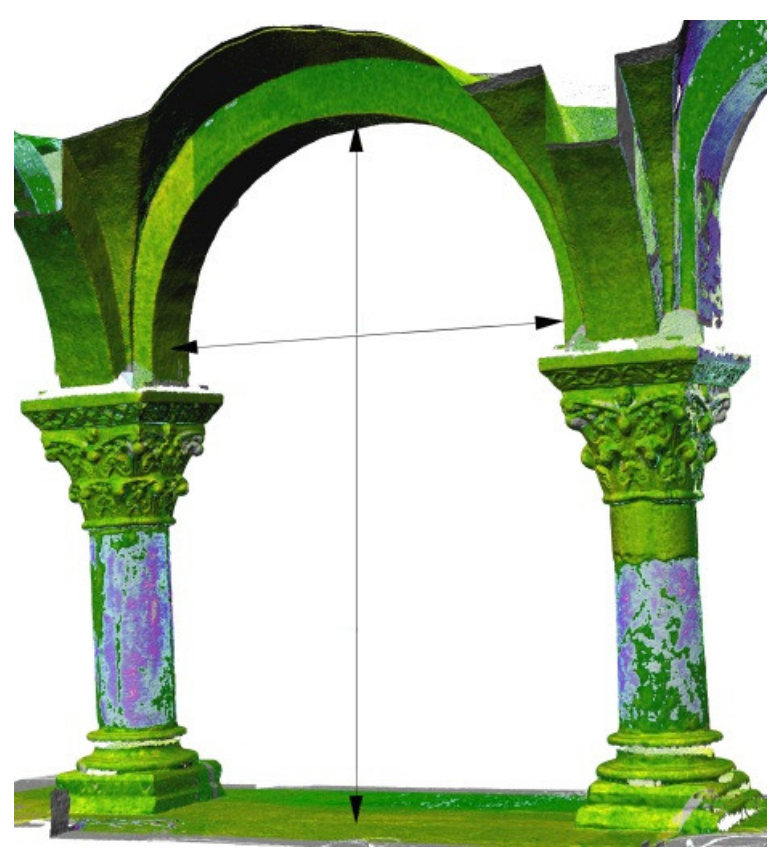

Figure 16. Dimensional inspection 


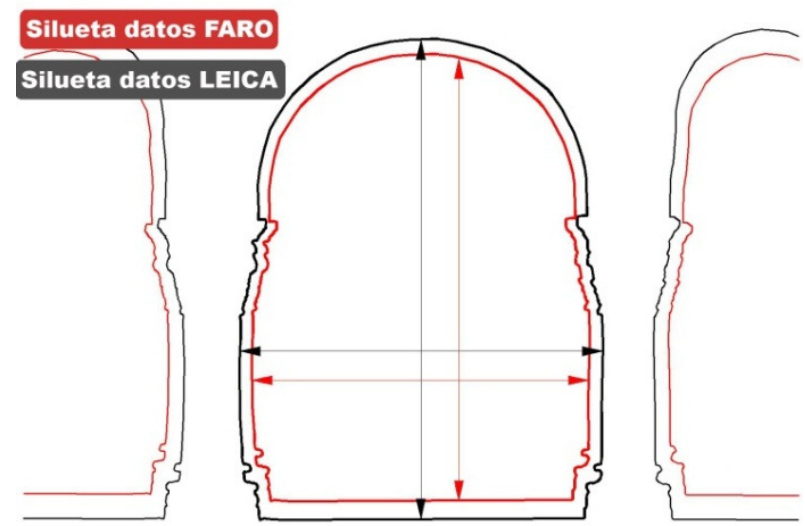

Figure 17. Significant scale differences have been found.

Automatic alignment obtained by "Best fit", despite introducing less uncertainty when superimposing both point clouds, performs the process calculating the average among all the composing parts of the object, therefore the alignment will be determined by a function which simulates the correspondence between the centroids of two clouds with similar data. In this way the scale mistake performance cannot be studied through the whole scanning process, which could include an enlargement or reducing factor or present divergences between the horizontal and vertical components. The observed scale error is $0.358 \%$, representing a reduction of $3.58 \mathrm{~mm}$ per actual meter on the scene. There also were documented differences of $1,5 \mathrm{~mm}$ for the same capture in the domes of nearly $4.18 \mathrm{~m}$ height.

This scale difference phenomenon is related to an internal issue of the scanner, probably due to the equipment calibration. Laser scanners are accurate devices, with much optical, micromechanical and electronic complexity. Continuous use implies the exposure to dust, humidity, vibrations, impacts... as well as to the wear of mirrors and prisms because of the own hit of the laser beam. All of this contributes to reduce the proper operation of the equipment, hence the need for periodic calibration by specialists that are technologically qualified to perform these operations. Leica ScanStation C10 scanner was calibrated by the own Leica Geosystem firm shortly before performing the documentation of the Pantheon, whereas Faro scanner has not been calibrated since more than a year (In addition, the owner of the scanner reported to have detected some deficiencies in the operation occurred around the time of this experiment). This suggests that the scale error is due to the Faro Photon 80 scanner, because of its lack of calibration.

\subsection{Abrupt cut in continuous surfaces}

A mechanical component exists in most of the measurement instruments of this kind, adjustable manually in several occasions, which will have by definition limited guarantees of accuracy, introducing errors if it is not correctly gauged or it is not proven its exact position. The Faro Photon Scanner, contrary to the Leica $\mathrm{C} 10$, needs some manual operations to be done for every scan to put the imaging nodal point in the place of the rotating mirror centre.

The dslr camera is mounted on top of the scanner head but decentred to allow the laser vertical rotation during scans. The unbalanced weight of the camera might cause some torque on the tripod column that result in a sort of precession of the rotation axis.

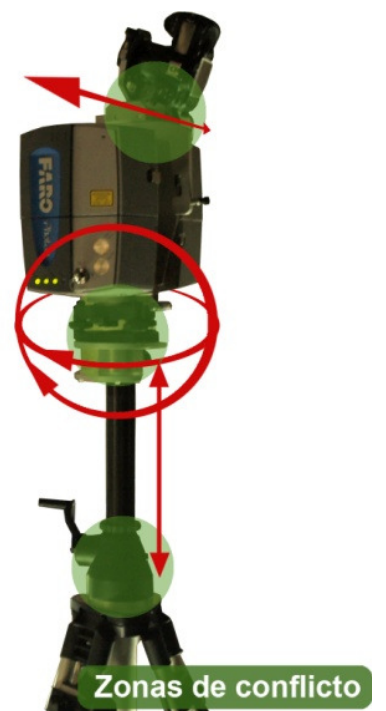

Figure 18. Possible weak points in the Photon setup.

The geared column clamp might not be tight enough to fully avoid any vertical displacement or even tilt of the whole set.

The levelling tribrach or the fixing bolt might have been causing small gap jumps during rotation of an unbalanced head

The mentioned manual operations to move the camera centre by means of lowering the whole scanner and the lateral displacement which is achieved by the sliding camera mount, can for sure affect the texture mapping quality, but also can have impact in mechanical parts that have not been designed to provide enough rigidity and repeatability. The abusive use of these moving parts produces wear and looseness which will eventually affect the geometric quality of the work. The Royal Pantheon point cloud documented with the Faro Photon 80 Laser Scanner presents an abrupt cut in one of the tracts of the head, in form of vertical profile, affecting the geometric continuity of the head wall face as well as the interior dome arch.

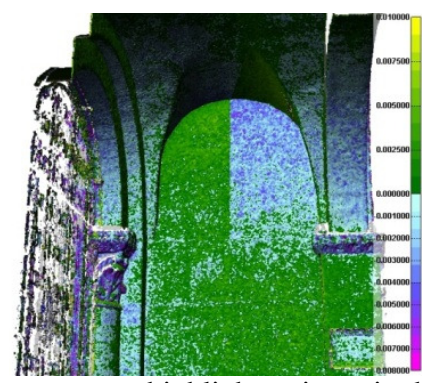

Figure 19. The error map highlights a jump in the Photon data.

The discontinuity presents an inflection point in the edge that divides the wall face and vault, happening between the vertical quadrant and the horizontal one, a way change in the vectors of displacement normal for the compared clouds. This event answers to a possible translation and draft of the scanner laser equipment with regard to his initial position, happening a change of the reference point, fundamentally in the $\mathrm{X}$ and $\mathrm{Z}$ axes, for what the displacement or "nod" will have different components in both axes. The maximum of this gap on the wall face is of $0.013 \mathrm{~m}$ while, on the flying buttress (vertical quadrant) it is $0.008 \mathrm{~m}$. This estimation takes incorporated the scale error between the scanners described in the section 6.2. 


\section{CONCLUSION}

Increasingly necessary precision in the documentation of the cultural tangible heritage for the wide range of interventions that we must attack forces (at the same time that impels) the development of instruments with major facilities. The present study documents the test of the terrestrial laser scanners on a real case and compares the geometric accuracy of the information obtained across the Faro Photon 80 and Leica ScanStation $\mathrm{C} 10$ on the same scene.

Even though are based on different principles of operation, the accuracy specs given by the manufacturers and the capture setting for the present example are very similar. For this reason the comparisons between the output of each of them, should throw values with minimal variations, which has not happened, on the contrary variations have been documented by the order of the dozens of millimetres.

The comparative study and the investigation of possible reasons behind differences lead us to determining that the Faro Photon Laser Scanner achieves less absolute accuracy compared to the Leica ScanStation C10 which on the other side is slightly less capable of resolving very small details. Arguably phase-shift scanners are profiled as the technology of the future for architectural heritage scenario, while pure time-of-flight technology might have found its physical limits. Having said that, we have verified that, a last generation TOF gun, can successfully compete and even surpass the not-so-new but still up-to-date PS technology present in the Faro Photon80

\section{ACKNOWLEDGEMENTS:}

Our thanks to Leica for providing the scanner and the technician Alberto Bushell for his invaluable help with the scans. The work in the Pantheon of Kings in San Isidoro has been carried out by researchers of the Architectural Photogrammetry Laboratory from the E.T.S. Arquitectura, Universidad de Valladolid, in collaboration with the architects Carlos Sexmilo and Ramón Cañas, both from León. It has been financed by the MICINN (Spanish Ministry of Science and Innovation) within the ADISPA project BIA2009-14254-C0201BIA2009-14254-C02-01. With the participation of the teachers Javier Finat Codes, Juan José Fernández Martín and Jesús San José Alonso, the Laboratory technician José Martínez Rubio, the research fellow Jorge García Fernández and the research staff: Luis García García, David Marcos González, Francisco Morillo Rodríguez and Mónica Ruiz Rituerto. 